\title{
Modelling Network Interdependencies of Regional Economies using Spatial Econometric Techniques'
}

\author{
Péter Járosi Multiregional dynamic models of economic \\ Regional Research Institute growth rarely capture the interdependencies \\ West Virginia University among regions that are geographically distant \\ E-mail: and/or often underestimate the importance of \\ peter.jarosi@mail.wvu.edu these linkages. This bias has become more and \\ more serious because travel and transportation \\ costs continue to decrease, while new telecommu- \\ nication and information technologies enable \\ business activities to readily take place between \\ geographically remote locations. The conceptual \\ framework in this study-modelling the network of \\ regions-is based on well-known spatial economet- \\ ric methods and provides alternative ways to inte- \\ Keywords: grate network interdependencies of economic ac- \\ spatial econometrics, tivities into many fields, as well as modelling tech- \\ input-output analysis, \\ network of regions, \\ interregional trade \\ niques such as spatial computable general equilib- \\ rium, input-output, and dynamic econometric \\ models.
}

\section{Introduction}

Both spatial econometric models and network-based regressions can be considered as special cases of cross-sectional dependence regressions, where the relationships between regions are defined by spatial proximity and the edges of the network structure, respectively. LeSage and Debarsy (2016) suggest replacing the word 'spatial' with the term 'cross-sectional dependence' when weight matrices are not created through spatial proximity, but via any other non-spatial proximity definition. In this article, I take the example of interindustry relationships to show how further development of these techniques is possible based on weight matrices that are created not only by contiguity and spatial distance algorithms, but also by defining network distance measurements. Empirically, long-distance cross-sectional dependencies can be more important than spatial ones depending on the industry; nevertheless, there are still many traditional industries where spatial autocorrelation can be dominant while other cross-sectional dependencies remain insignificant.

\footnotetext{
${ }^{1}$ This paper was presented at the $56^{\text {th }}$ Meeting of the Southern Regional Science Association, Memphis, Ten-
} nessee, April 2017.

Regional Statistics, Vol. 7. No.1. 2017: 003-016; DOI: 10.15196/RS07101 
For regional policy, it is essential to: identify the key industries within a given region and analyse their spatial and network dependencies, including geographically distant linkages; measure their dependencies from weaker to stronger; and know how to influence them to improve their economic performance. Before making policy decisions, it is crucial to run simulations using dynamic models, implement regional impact assessment, and take into consideration interregional and interindustry effects beyond macroeconomic aspects and regional impacts. In this respect, the main goal of this study is to provide theoretical and methodological insights about the importance of regional network interlinkages of key industries, as well as a proof of concept for future network econometrics and economic modelling research.

\section{Problem definition}

The aim of this study is to shed light on an alternative conceptual framework to build dynamic multiregional economic models in general, and to develop a new method to connect regions within multiregional models, as well as to emphasise the advantages of the new approach in the modelling of interregional and interindustry linkages in particular. The approach is based on the creation econometric equations (stochastic equations) among the spatial units instead of using identities (deterministic equations) and can be applied to many other fields other than economics.

Several methods enable to connect regions within an economic model, and it is usually difficult to find the appropriate method depending on the purpose of the model. One of the most popular approaches is based on the well-known gravity model, where the intensity of the relationships decreases as geographical distance increases. In many cases, this approach provides the most satisfactory solution; thus, it is widely accepted, and this aspect of the multiregional model is rarely questioned or criticised. In some cases, if the model considers a more detailed level of industrial classification, distant interdependencies can become more important than the ones across closer neighbours, especially in industries where transportation costs are relatively low compared to the value of the transported goods. Here, the gravity model cannot provide accurate results, as it underestimates the economic interactions among regions, which can be physically far apart. Nonetheless, in other industries, where spatial proximity does matter, the gravity model can still be the best solution. The problem occurs, for example, between regions where the gravity model predicts low trade value, although they actually exhibit strong interdependency for a given industry. There are many industry-specific examples for this phenomenon, some of which are outlined below.

Spatial econometrics provides stochastic equations for multiregional economic models to capture the interactions among nearby regions. By connecting regional variables in many different regions, these equations allow modellers to establish interregional linkages for dynamic simulations. This method can be interpreted as an

Regional Statistics, Vol. 7. No.1. 2017: 003-016; DOI: 10.15196/RS07101 
extension of spatial econometric techniques where the weight matrix generates from network linkages among regions instead of traditional definitions (binary contiguity, inverse distance, etc.). In this study, the network definition is based on interregional trade data, although this is not the only way to create alternative types of crosssectional weight matrices. Depending on the investigated phenomena, many other matrix representations of directed or undirected graphs of interregional connections are imaginable and applicable in order to build weight matrices.

In this context, the word 'network' refers to the network of regions. However, generally speaking, the word has a much wider meaning (e.g. social networks, neural networks, computer networks, etc).

Considering whether the framework of network theory can be employed to describe the interregional interindustry linkages, Rodrigues et al. (2016) illustrate the case of an input-output model in which the network approach can result in much clearer and more flexible modelling techniques than conventional matrix formulations. Additionally, they point out the advantages of the topological transformations applied to these network structures.

Cross-sectional dependence seems to be a broader concept than network of regions, as it involves many other applications that cannot be described in terms of network model.

\section{Examples of network-based weight matrices}

The following examples provide the base for a proof of concept of a multiregional model, which will be developed in the next step of this research. Network autocorrelation can usually be defined similarly to spatial autocorrelation.

The idea of defining network autocorrelation and creating weight matrices by network structures and topologies is not new and has already been accepted in social network studies. Leenders (2002) provides a clear definition: 'social influence enters network autocorrelation models through the weight matrix $\mathrm{W}$, also called the structure matrix. Entry $\mathrm{w}_{\mathrm{ij}}$ represents the extent to which $\mathrm{y}_{\mathrm{i}}$ is dependent on $\mathrm{y}_{\mathrm{i}}$ thus to what extent actor $j$ influences i'. Doreian (1989) describes the mathematics of different types of network models (network effects, network disturbances, and their mix) that can be employed to investigate network effects on social actors. Although an overview about the methodology of weight matrices of social networks can be obtained, the cross-sectional dependence of the interregional input-output linkages seems to be a different problem. For instance, the problem size is much smaller than in the case of social networks, which is important for the algorithms running on computers. Inverting a ten-million by ten-million sized matrix seems to be difficult and CPU time consuming; nonetheless, nowadays it is not impossible, and fortunately it is not even necessary in the case of the network of regions because of the limited number of nodes.

Regional Statistics, Vol. 7. No.1. 2017: 003-016; DOI: 10.15196/RS07101 
There are software packages allowing users to generate network spatial weights. For example, in ArcMap it is possible '...to model and store spatial relationships based on time or distance between point features in the case where travel is restricted to a network dataset' (ArcMap 2017). Moreover, PySal contains functions for network constrained analysis (Rey 2016).

Some contributions in the literature apply a combination of the network and the spatial approaches; for example, Ermagun and Levinson (2016) introduce the network weight matrix as a replacement for the spatial weight matrix. Specifically, with the aim of modelling traffic flows, they define the elements of the weight matrix by network topology and structure beyond spatial proximity.

In many cases, it is possible to reduce the specification of the econometric model into a simpler estimation and inference, in order to still apply conventional techniques and software packages of spatial econometrics. LeSage (2017) develops an algorithm based on convex combinations of matrices to redefine the specification of the cross-sectional dependence regression replacing two or more different weight matrices by a single weight matrix. The latter combines all original matrices with their correspondent importance.

In the simplest version of the multiregional model suggested in this study, the equations of the network regression models can be written similarly to the ones of the spatial regression models; for example, the equation of the Network Autoregressive Model (NAR) is almost the same as the equation for the Spatial Autoregressive Model (SAR), and only the definition of the weight matrix is different:

$$
y=\varrho W y+X \beta+\varepsilon
$$

Furthermore, in most cases the estimation methods of the spatial econometric models are also applicable to the network econometric models. Moreover, the usage of the available software packages (Geoda, R, PySal, etc.) is possible, provided that there is a flexibility to rewrite the weight matrices. For the present application, it was convenient to use the weight matrix object and the spreg.ml_lag function of the PySal package in a Python script. This 'module provides spatial lag model estimation with maximum likelihood following (Anselin 1988)', as quoted by Rey in the software documentation (Rey 2016). ${ }^{2}$

Because it is only a conceptual description of the alternative approach, my example consists of a simple econometric model to prove the existence of this type of network autocorrelation. To illustrate it, an apparent relationship between the compensation per employee and labour productivity measured by gross industrial product per employee is assumed. Both variables are generated in a two-dimensional state by industry tables for year 2012. In this example, the states are chosen as spatial units; thus, the word 'region' will be used as a synonym for 'state'.

${ }^{2}$ pysal.readthedocs.io/en/latest/library/spreg/ml_lag.html (downloaded: March 2017)

Regional Statistics, Vol. 7. No.1. 2017: 003-016; DOI: 10.15196/RS07101 


\section{Data}

Data on employment, compensations, gross industrial product, input-output accounts, and coefficients for 51 states and 71 industries were retrieved directly from the IO-Snap software, a third-party commercial data vendor. ${ }^{3}$ IO-Snap extracts the original tables from the U.S. Bureau of Economic Analysis (BEA) website, while facilitating the aggregation and regionalisation of the tables. The use of IO-Snap was more convenient than downloading data directly from BEA and creating a new preprocessing algorithm.

The interregional trade data are from the 2012 Commodity Flow Survey Public Use Microdata File (CFS PUMS file) ${ }^{4}$, which is downloadable from the United States Census Bureau website. Among the other things, this data file contains columns for the FIPS state code of shipment origin, the FIPS state code of shipment destination, and the NAICS code of the shipper; therefore, trade classification by industry and the origin state was also considered. In this example, regions are defined as states, but smaller or larger spatial units can also be used depending on the available data and the purpose of the analysis. The CFS PUMS file also provides information about the metro area of shipment origin, the CFS area of shipment origin, the metro area of shipment destination, the CFS area of shipment destination, and a two-digit SCTG commodity code of the shipment. By investigating the rows of the CFS PUMS file, it seems obvious that the trade matrix can also be generated for smaller spatial units. Moreover, beyond the industry by industry (IxI) analysis, commodity by commodity (CxC) and industry by commodity (IxC) analyses could also be implemented, because IO-Snap can easily use its regionalisation features to generate the necessary data. For example, the regionalisation process can be run for input-output accounts (final demand, value added, use and make tables) and tables of direct and total requirements in all three formats: IxI, IxC, and $\mathrm{CxC}$. The use of a CFS PUMS file is only an example of how to create weight matrices; indeed, there are many other possible ways to collect data and develop algorithms generating weight matrices to capture the far distance linkages. For example, regional level aggregations of researchers' social network can provide the representation of the interregional innovation linkages in a simple region by region cross-sectional weight matrix format.

\section{Generating network-based weight matrices}

Aggregating the microdata from the commodity flow survey produces industryspecific state-by-state tables of shipment values for each available NAICS code. Within a given industry, this matrix represents a directed graph where the nodes are

${ }^{3}$ www.io-snap.com (downloaded: March 2017)

${ }^{4}$ www.census.gov/econ/cfs/pums.html (downloaded: March 2017)

Regional Statistics, Vol. 7. No.1. 2017: 003-016; DOI: 10.15196/RS07101 
regions and the arcs are links defined by the following condition: two regions are connected if the total value of the shipment between them is greater than a given threshold. Any threshold is arbitrary, and one of the common choices is to specify the largest value, in order for all regions to have at least one neighbour. This algorithm results in a non-symmetric matrix of first-order neighbours. The higher-order neighbours in the network case can also be calculated through a method similar to binary contiguity matrices in spatial econometrics. Using the graph theory terminology, this is equivalent to the condition of the shortest path between two nodes. The non-symmetric weight matrix, that is, the directed graph, can be employed for exploratory data analysis (e.g. calculate Moran's I in the network case) and to derive the network lagged independent variables, especially for industries' backward and forward linkages-not only for the intraregional supply chain linkages, but also for interregional trade.

In order to run a cross-sectional dependence autoregressive model, a symmetric weight matrix is required. This is derived from the original shipment matrix by adding the matrix to its transpose (to add state $\mathrm{A} \rightarrow$ state $\mathrm{B}$ shipments to state $\mathrm{B} \rightarrow$ state $\mathrm{A}$ shipments and vice versa). This aggregation provides a symmetric matrix, but can cause an information loss in terms of shipment direction. Nevertheless, both directions can influence the interdependencies between two regions. Therefore, the result of the model might not be misleading. A possible improvement could deal with the development of a methodology of estimation and inference with asymmetric weight matrices; in other words, a way to handle directed graphs representing network structures in econometric models.

In the first version of the network-based weight matrix (Figure 2), the rows of the row-standardised matrix are unweighted; this means that the actual shipment value is ignored, and only the existence of connections is considered using the same weight for each neighbour. In the second version, different weights are assigned to the neighbours distinguished by the shipment value. The significance of the autocorrelation and the autoregressive coefficients does not substantially change between the two non-spatial models.

The neighbours' pattern in the network case is different from the spatial case. The first pair of maps (Figure 1) illustrates that the geographically defined neighbours surround the examined states.

In the second pair of maps (Figure 2), a very different pattern of neighbours can be seen for the same states. With the network-based method, some distant states became connected, while some spatial neighbours lost their connections to the given state.

The comparison of the four maps shows that an interesting outcome might occur. There are no joint neighbours in the examined two states in the spatial case because of the far distance; however, in the network-based case, they have one common neighbour. After defining the weight matrix by trade data, physically dis-

Regional Statistics, Vol. 7. No.1. 2017: 003-016; DOI: 10.15196/RS07101 
tant regions can become connected, such as Vermont and Oregon on the right-hand side of the map, while spatially connected neighbours might remain neighbours or become unconnected.

Figure 1

Spatial neighbours of Montana and Vermont using threshold of distance method

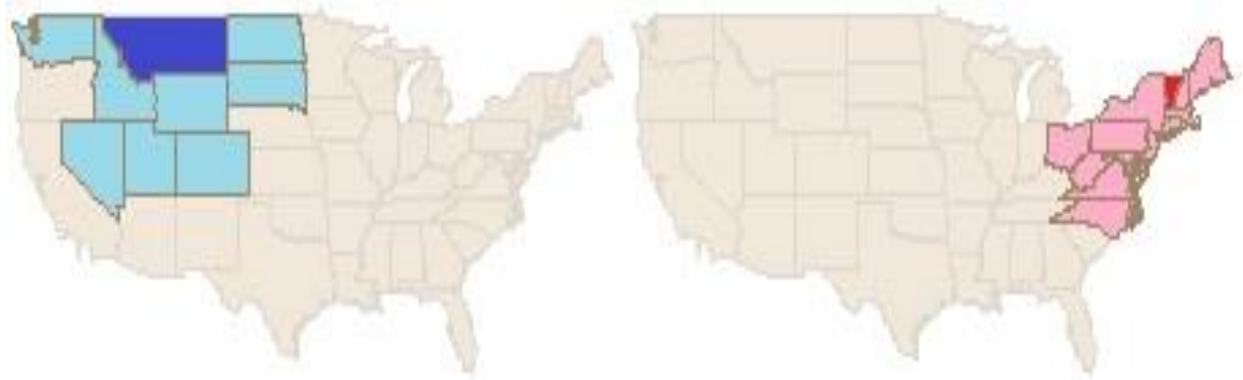

Source: Generated using GeoDa v1.8.16.4; 1 March 2017, coloured manually.

Figure 2

Network-based neighbours of Montana and Vermont by trade in industry NAICS code 332, Fabricated Metal Product Manufacturing

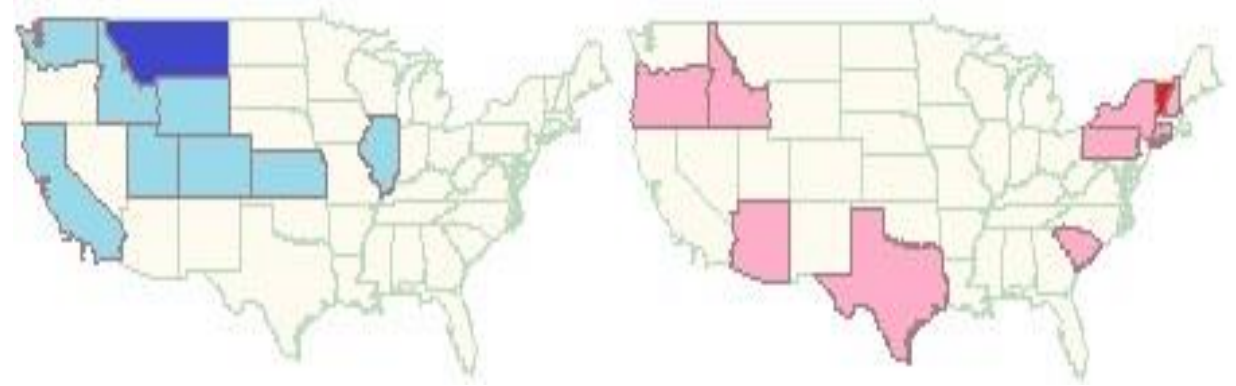

Source: Generated using GeoDa v1.8.16.4; 1 March 2017, coloured manually.

\section{Empirical results}

Based on a simple example, one spatial model (with binary contiguity weight matrix) and two network models (network threshold type and network flow type) are created for each industry for which interregional trade data are available. The equation of the spatial autoregressive model (Anselin 1988) is as follows:

$$
y=\varrho W_{S} y+a+\beta_{1} x_{1}+\left(\beta_{2} x_{2}+\ldots+\beta_{k} x_{k}\right)+\varepsilon,
$$

where the dependent variable $y$ represents the compensation rates by states within a given industry, $W$ is the same binary contiguity spatial weight matrix for each industry, and $x_{1}$ is the independent variable of labour productivity by states within a given industry. Although it would possible to use more explanatory variables $x_{2} \ldots x_{k}$, in this simple example I use only one. Thus, in order to simplify the notation, $x$ will

Regional Statistics, Vol. 7. No.1. 2017: 003-016; DOI: 10.15196/RS07101 
replace $x_{1}$ henceforth. Actually, equation (2) is a generalised equation that can be written for each industry $i$.

First, spatial autoregressive models are implemented for each industry from IO code 212 to 55, and the cases of positive significant autoregressive parameter are listed in Table 1. In many cases, the autoregressive parameters become significant, and the spatial diagnostic of OLS estimations show more robust Lagrange multipliers for the spatial lag model than for the spatial error model (in the significant cases).

Table 1

The cases of positive significant spatial autoregressive parameter

\begin{tabular}{c|l|c|c|c|c|c}
\hline $\begin{array}{c}\text { IO } \\
\text { code }\end{array}$ & \multicolumn{1}{|c|}{ Name } & $\begin{array}{c}\varrho \\
\text { coefficient }\end{array}$ & $\begin{array}{c}\varrho \\
\text { probability }\end{array}$ & $\begin{array}{c}\beta \\
\text { coefficient }\end{array}$ & $\begin{array}{c}\beta \\
\text { probability }\end{array}$ & $\begin{array}{c}\text { Spatial } \\
\text { pseudo R }\end{array}$ \\
\hline 321 & Wood products & 0.3097636 & 0.0000031 & 0.3314931 & 0.0000000 & 0.7471041 \\
331 & Primary metals & 0.3827188 & 0.0000633 & 0.0327501 & 0.0110314 & 0.1577813 \\
333 & Machinery & 0.2343926 & 0.0266329 & 0.2006220 & 0.0000623 & 0.2931058 \\
335 & Electrical equipment, appliances, components & 0.2445147 & 0.0083023 & 0.2290565 & 0.0000000 & 0.4491828 \\
337 & Furniture and related products & 0.2547117 & 0.0027271 & 0.2847682 & 0.0000000 & 0.5247478 \\
339 & Miscellaneous manufacturing & 0.2807512 & 0.0143612 & 0.2362123 & 0.0000000 & 0.6514770 \\
323 & Printing and related support activities & 0.2422890 & 0.0051829 & 0.2539650 & 0.0000033 & 0.3709606 \\
325 & Chemical products & 0.4077508 & 0.0036684 & 0.0792176 & 0.0000230 & 0.3231142 \\
326 & Plastics and rubber products & 0.2209095 & 0.0018884 & 0.1839768 & 0.0000003 & 0.5240715 \\
42 & Wholesale trade & 0.1469258 & 0.0172730 & 0.4867514 & 0.0000000 & 0.7071403
\end{tabular}

In the second step, the network lagged variables are created similarly to the spatially lagged variables, the only difference being the weight matrices' method. In the network-based case, I employ two types of row-standardised network matrices, while the other parts of the algorithm are mathematically equivalent to the spatial case. Thus, equation (2) can be rewritten as:

$$
y_{i}=\varrho W_{N, i} y_{i}+a+\beta x_{i}+\varepsilon \text {. }
$$

In equation (3), $y_{i}$ and $x_{i}$ are still vectors for all industries $i$, and the elements of such vectors are the values of dependent and independent variables by region, respectively.

The notation is almost the same as in equation (2), except that the weight matrices are network-based instead of spatial matrices; and per definitionem, they are different for each industry depending on the interregional trade data.

The results in Table 2 generate from the unweighted network-connectivity matrices, while the corresponding results of the weighted network-flow matrices are showed in Table 3. All industries have different weight matrices in both cases (compared to the other industries) according to the total trade value by industry among the states.

Regional Statistics, Vol. 7. No.1. 2017: 003-016; DOI: 10.15196/RS07101 
Table 2

The cases of positive significant network autoregressive parameter using network threshold type weight matrices

\begin{tabular}{c|l|c|c|c|c|c}
\hline $\begin{array}{c}\text { IO } \\
\text { Code }\end{array}$ & \multicolumn{1}{|c|}{ Name } & $\begin{array}{c}\varrho \\
\text { coefficient }\end{array}$ & $\begin{array}{c}\varrho \\
\text { probability }\end{array}$ & $\begin{array}{c}\beta \\
\text { coefficient }\end{array}$ & $\begin{array}{c}\beta \\
\text { probability }\end{array}$ & $\begin{array}{c}\text { Spatial } \\
\text { pseudo R }\end{array}$ \\
\hline 332 & Fabricated metal products & 0.7359775 & 0.0000032 & 0.3743817 & 0.0000000 & 0.6980953 \\
337 & Furniture and related products & 0.5951258 & 0.0029552 & 0.3245427 & 0.0000000 & 0.4780375 \\
323 & Printing and related support activities & 0.6899227 & 0.0000390 & 0.2942893 & 0.0000000 & 0.3252263 \\
326 & Plastics and rubber products & 0.6577262 & 0.0016704 & 0.2150794 & 0.0000000 & 0.4991782
\end{tabular}

Table 3

The cases of positive significant network autoregressive parameter using network flow type weight matrices

\begin{tabular}{c|l|c|c|c|c|c}
\hline IO Code & \multicolumn{1}{|c|}{ Name } & $\begin{array}{c}\varrho \\
\text { coefficient }\end{array}$ & $\begin{array}{c}\varrho \\
\text { probability }\end{array}$ & $\begin{array}{c}\beta \\
\text { coefficient }\end{array}$ & $\begin{array}{c}\beta \\
\text { probability }\end{array}$ & $\begin{array}{c}\text { Spatial } \\
\text { pseudo R }\end{array}$ \\
\hline 332 & Fabricated metal products & 0.6186635 & 0.0007193 & 0.3723363 & 0.0000000 & 0.6798423 \\
$3364 \mathrm{OT}$ & Other transportation equipment & 0.4214841 & 0.0250983 & 0.2114154 & 0.0000000 & 0.3346656 \\
337 & Furniture and related products & 0.7259708 & 0.0000026 & 0.3320034 & 0.0000000 & 0.5111702 \\
323 & Printing and related support activities & 0.7390262 & 0.0000001 & 0.2676689 & 0.0000003 & 0.3494023 \\
326 & Plastics and rubber products & 0.8205607 & 0.0000000 & 0.2113470 & 0.0000000 & 0.5243172
\end{tabular}

Network autocorrelation occurs less frequently than spatial autocorrelation. In a few cases, especially in the fabricated metal products industry, network interdependencies are observed (irrespective of which type of network matrices is applied), despite the spatial autoregressive parameter is not significant. As expected, the coefficients of the explanatory variables are positive and significant in all cases, because labour productivity can explain the variance of compensations reasonably well. The PySal report summary for both network cases can be seen in Tables 4 and 5 .

The conditions of the two network autoregressive models reported in Tables 4 and 5 only differ for weight matrices; net_thres and net_flow denote binary network threshold type and weighted network flow type, respectively. The results from the two types of network weight matrices do not emerge as very different.

The third step is the extension of the network autoregressive model in equation (3) to intraregional backward linkages. The interindustry weighted explanatory variable generates from the national direct requirement table:

$$
y_{i}=\varrho W_{N, i} y+a+\beta x_{i}+\gamma W_{B} x_{r}+\varepsilon .
$$

Note: in equation (4), vectors $x_{i}$ and $x_{r}$ are not in the same structure; nonetheless, they represent the same explanatory variable (labour productivity). Vector $x_{i}$ contains the values of regions within a given industry, and vector $x_{t}$ contains the values of industries in a given region. The transformations in $W_{B}$ are implemented in all regions; thus, these lagged variables are in the same structure as the original $x_{i}$ variables and could be handled as regular independent variables.

Regional Statistics, Vol. 7. No.1. 2017: 003-016; DOI: 10.15196/RS07101 
Table 4

Results of Network Autoregressive Model for NAICS 332, Fabricated Metal

Product Manufacturing, using network threshold type weight matrix

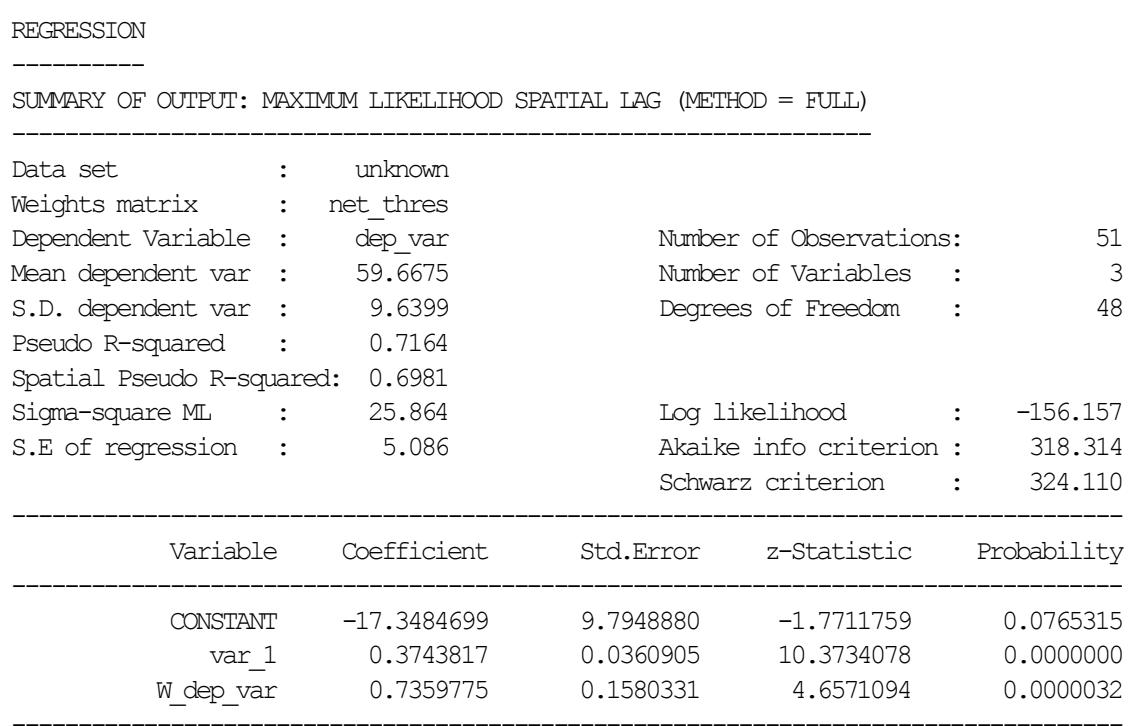

Results of Network Autoregressive Model for NAICS 332, Fabricated Metal

Product Manufacturing, using network flow type weight matrix

REGRESSION

SUMMARY OF OUTPUT: MAXIMUM LIKELTHOOD SPATIAL IAG (METHOD = FULL)

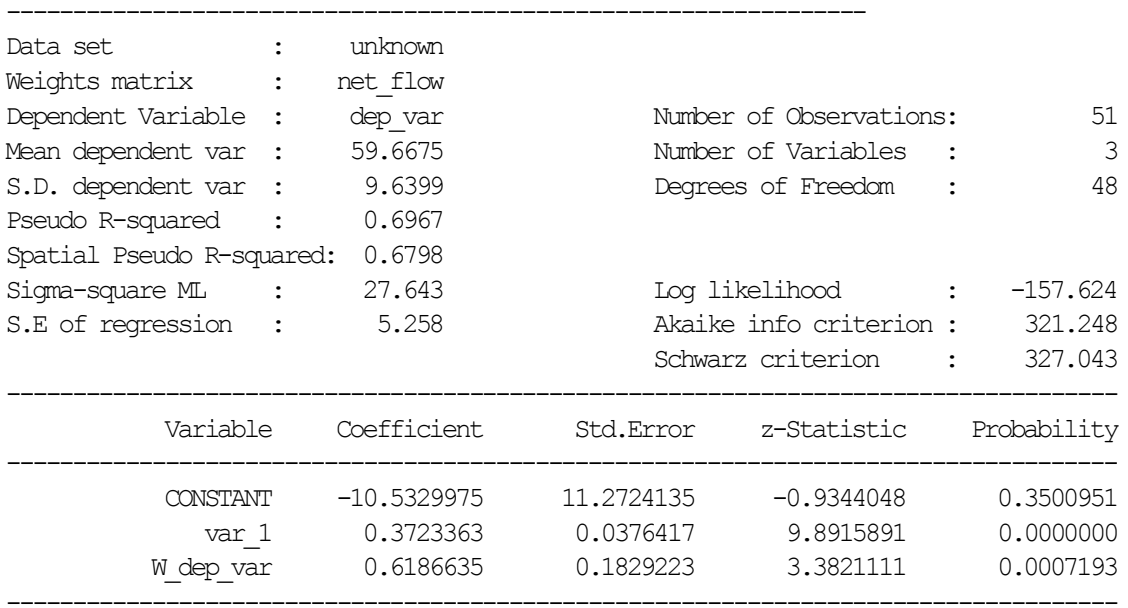

Regional Statistics, Vol. 7. No.1. 2017: 003-016; DOI: 10.15196/RS07101 
The explanatory variable (within a given region) is weighted by the coefficients from the columns of the direct requirement table of the industry-by-industry structure, excluding the examined industry itself. There are three transformations between the matrix $W_{B}$ and the original industry-by-industry table: replacing the main diagonal of the input-output table with zeros, transpose it, and the rowstandardisation. This new variable in equation (4) can be considered as an explanatory variable for backward linkages. Matrix $W_{B}$ has no index $r$, this means that the national input-output table is used in the first approximation, but there are options in IO-Snap to regionalise the table of direct requirements and replace $W_{B}$ matrix with different regional $W_{B, r}$ matrices for each region.

Table 6

Results of the network autoregressive models with backward linkages

\begin{tabular}{c|c|c|c|c|c|c|c}
\hline IO Code & $\begin{array}{c}\varrho \\
\text { coefficient }\end{array}$ & $\begin{array}{c}\varrho \\
\text { probability }\end{array}$ & $\begin{array}{c}\beta \\
\text { coefficient }\end{array}$ & $\begin{array}{c}\beta \\
\text { probability }\end{array}$ & $\begin{array}{c}\text { Spatial } \\
\text { pseudo R }\end{array}$ & $\begin{array}{c}\gamma \\
\text { coefficient }\end{array}$ & $\begin{array}{c}\gamma \\
\text { probability }\end{array}$ \\
\hline 212 & -0.2068741 & 0.1738937 & 0.0877126 & 0.0000000 & 0.4117533 & 0.0451872 & 0.0172671 \\
327 & 0.4593082 & 0.0420149 & 0.1537810 & 0.0007127 & 0.2416895 & 0.0309428 & 0.1032600 \\
332 & 0.6407994 & 0.0003017 & 0.3621068 & 0.0000000 & 0.6934640 & 0.0189350 & 0.1391610 \\
333 & 0.3286246 & 0.2139013 & 0.1944382 & 0.0000760 & 0.3505849 & 0.1272176 & 0.0096165 \\
334 & -0.1889946 & 0.5149651 & 0.0715192 & 0.0000050 & 0.3450205 & 0.2490942 & 0.0041148 \\
337 & 0.7469666 & 0.0000002 & 0.2953514 & 0.0000000 & 0.5151907 & 0.0574779 & 0.1666706 \\
$315 \mathrm{AL}$ & 0.2018436 & 0.3359634 & 0.2917739 & 0.0000000 & 0.6145860 & 0.1577364 & 0.0014496 \\
323 & 0.7860992 & 0.0000000 & 0.2602953 & 0.0000002 & 0.3492147 & 0.0312487 & 0.0347412 \\
326 & 0.8192370 & 0.0000000 & 0.1940023 & 0.0000000 & 0.5505027 & 0.0149876 & 0.0536604 \\
42 & 0.0655316 & 0.7516733 & 0.2917696 & 0.0000444 & 0.7508864 & 0.2056734 & 0.0002273
\end{tabular}

The backward linkages parameter explains the effect of changes in labour productivity in the supplier industries on compensations in the considered industry.

Given this, a possibility is to estimate the econometric equations with a multidimensional weight matrix defined as a combination of interregional and interindustry linkages:

$$
y=\varrho W_{N} y+a+\beta x+\gamma W_{B} x+\delta\left(W_{N} \times W_{B}\right)_{x}+\varepsilon .
$$

The notation $\times$ between $W_{N}$ and $W_{B}$ is not the regular matrix multiplication. Instead, this transformation results in a three-dimensional weight matrix from the two-dimensional matrices, and can be defined by the elements of the original matrices using the following formula. Specifically, denote $z_{i, r}$ the elements of the new variable in industry $i, n_{t, s, j}$ the elements of matrix $W_{N}$ in industry $j, b_{i, j, r}$ the elements of matrix $W_{B}$ in region $r$, where indices $r, s$ represent the regions ( $s=$ origin, $r=$ destination), while indices $i, j$ denote related industries $(j->i)$ in the supply chain:

$$
z_{i, r}=b_{i, j, r} n_{r, s, j} x_{j, s}
$$

Regional Statistics, Vol. 7. No.1. 2017: 003-016; DOI: 10.15196/RS07101 
The projections of the multidimensional weight matrix (into the interregional plane) are not symmetric; in other words, they represent the directed graphs of interregional backward linkages. Developing a methodology through which econometric equations are defined by multidimensional weight matrices will make it possible to examine the effects of interregional supply chain linkages in an econometric context.

\section{Dynamic multiregional modelling framework}

The simple econometric equations representing the stochastic relationship between compensations and labour productivity only aimed to illustrate the opportunities behind the network-based weight matrix definition. In dynamic multiregional models, more sophisticated equations and econometric models are needed. These new equations will define the interlinkages among the most important regional variables (TFP, labour, capital, factor prices, $R \& D$ expenditures, etc.) and create dynamic interregional dependencies for simulations.

The network-based econometric equations, created from the new type of weight matrices, connect the variables in the multiregional model and establish interdependencies among regions; however, several other types of interregional equations can be added to the model to run dynamic simulations. The regional social accounting matrices can be linked by using different types of equations: identities, gravity model, and stochastic equations (spatial, network-based, and other cross-sectional econometric models). The best solution seems to be a mix of different types of equations, especially when the network-based estimation is not significant or is not possible to implement because of the lack of data.

\section{Regional policy implications}

The impact assessment can be implemented by changing the initial values of the exogenous variables. In this way, the shocks added to the system are defined as the differences between new and original values of the variables. This technique represents a powerful tool to investigate the dynamics of the multiregional model responding to an economic shock.

The dynamic simulations highlight the consequences of changes in the policy variables, which can be implemented by using macroeconomic and multiregional models separately, or by developing a mixed macroeconomic-multiregional model. The multisectoral regional blocks of the suggested model can support the identification of key industries within regions, while distinguishing spatially and network dependent sectors. Different types of industries justify different policies, depending on the observed interregional, backward, and forward linkages.

Regional Statistics, Vol. 7. No.1. 2017: 003-016; DOI: 10.15196/RS07101 


\section{Conclusions and further research}

The methodology to create network-based regions' weight matrices has been outlined, and the existence of multiregional network autocorrelation has been proved through a simplified econometric example.

The construction of interregional linkages in the multiregional modelling framework via network and spatial econometric equations is more convenient than the burdensome creation of the identities to connect regions' social accounting matrices. Collecting reliable data for these problematic identities also seems to be laborious or sometimes even impossible. Moreover, the assumptions of the spatial computable general equilibrium modelling framework (e.g. short-term spatial equilibrium of the labour market) are not verifiable. In this new approach, the long-term dynamic simulation using the interregional econometric equations can provide results as reasonable as the ones from spatial computable general equilibrium models. As for the macroeconomic and the intraregional blocks of the integrated model, the computable general equilibrium approach and the identities creation through the social accounting matrix are still recommended. The most appropriate solution might be a mixed type of econometric, input-output, and computable general equilibrium model, where the interregional linkages are simulated by network, gravity, and spatial econometric equations replacing the conventional identities.

In the future, an alternative way to build a lightweight version of dynamic multiregional models will be developed by using the network econometrics equations.

\section{REFERENCES}

ANSELIN, L. (1988): Spatial Econometrics: Methods and Models. Kluwer Academic Publishers. Dordrecht.

ARCMAP v 10.3 (2017): A software to create maps, perform spatial analysis, manage geographic data, and share results. desktop.arcgis.com/en/arcmap/ (downloaded: March 2017)

DOREIAN, P. (1989): Models of network effects on social actors. In Research methods in social analysis, L. C. Freeman, D. R. White and K. Romney (Eds), George Mason University Press, Fairfax, pp. 295-317.

Ermagun, A.-Levinson, D. M. (2016): Development and Application of the Network Weight Matrix to Predict Traffic Flow for Congested and Uncongested Conditions. Retrieved from the University of Minnesota Digital Conservancy, hdl.handle.net/11299/181542 (downloaded: March 2017)

GEODA v1.8.16.4 (2017): A free and open source software tool that serves as an introduction to spatial data analysis. The Center for Spatial Data Science, University of Chicago. spatial.uchicago.edu/software (downloaded: March 2017)

IO-SNAP (2017): Input output - state and national analysis program. www.io-snap.com/ (downloaded: March 2017)

LEENDERS, RAJ. (2002): Modeling social influence through network autocorrelation: constructing the weight matrix. Social Networks 24: 21-47.

Regional Statistics, Vol. 7. No.1. 2017: 003-016; DOI: 10.15196/RS07101 
LeSAgE, James P.-Debarsy, N. (2016): Flexible dependence modeling using convex combinations of different types of weight structures. Manuscript. The paper has been submitted to Regional Science \& Urban Economics.

LESAGE, JAMES P. (2017): Mixing spatial and other types of cross-sectional dependence. Getis-Ord Lecture, Western Regional Science Association meetings, Santa Fe, New Mexico, February 2017.

REY, S. (2016). PySal v 1.13.0 An open source library of spatial analysis functions written in Python. pysal.readthedocs.io/en/latest (downloaded: March 2017)

Rodrigues, J.-MArques, A.-Wood, R.-Tukker, A. (2016): A network approach for assembling and linking input-output models, Economic Systems Research, 28:4, 518-538, DOI: 10.1080/09535314.2016.1238817

Regional Statistics, Vol. 7. No.1. 2017: 003-016; DOI: 10.15196/RS07101 